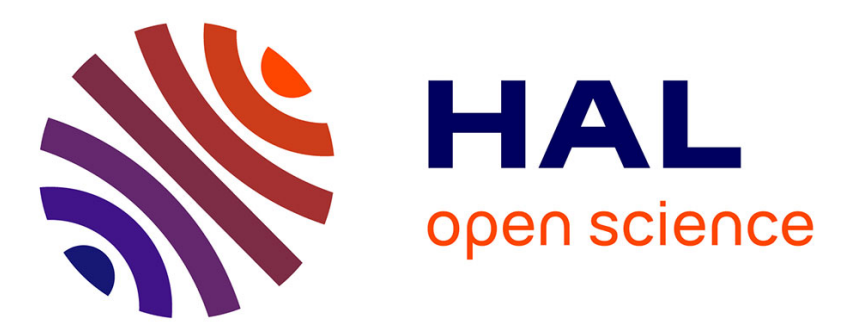

\title{
Oscillations d'intensité RHEED liées aux mécanismes de croissance de GaSb par E.J.M.
}

\author{
M. Nouaoura, C. Raisin, F.W.O. da Silva, M. Dumas, L. Lassabatère
}

\section{To cite this version:}

M. Nouaoura, C. Raisin, F.W.O. da Silva, M. Dumas, L. Lassabatère. Oscillations d'intensité RHEED liées aux mécanismes de croissance de GaSb par E.J.M.. Revue de Physique Appliquée, 1990, 25 (9), pp.915-921. 10.1051/rphysap:01990002509091500 . jpa-00246254

\section{HAL Id: jpa-00246254 https://hal.science/jpa-00246254}

Submitted on 1 Jan 1990

HAL is a multi-disciplinary open access archive for the deposit and dissemination of scientific research documents, whether they are published or not. The documents may come from teaching and research institutions in France or abroad, or from public or private research centers.
L'archive ouverte pluridisciplinaire HAL, est destinée au dépôt et à la diffusion de documents scientifiques de niveau recherche, publiés ou non, émanant des établissements d'enseignement et de recherche français ou étrangers, des laboratoires publics ou privés. 


\title{
Oscillations d'intensité RHEED liées aux mécanismes de croissance de GaSb par E.J.M.
}

\author{
M. Nouaoura, C. Raisin, F. W. O. Da Silva, M. Dumas et L. Lassabatere \\ Laboratoire d'Etudes des Surfaces, Interfaces et Composants (UA CNRS D07870), Université de Montpellier \\ II, Sciences et Techniques du Languedoc, Place Eugène Bataillon, 34095 Montpellier Cedex 5, France
}

(Reçu le 21 décembre 1989, révisé le 16 mars 1990, accepté le 9 mai 1990)

\begin{abstract}
Résumé. - Dans cet article, nous présentons une étude par la méthode des oscillations de l'intensité RHEED des mécanismes de croissance de GaSb par E.J.M. Nous avons précisé l'influence de différents paramètres (température du substrat, flux d'antimoine et de gallium, temps d'interruption de croissance) sur le régime oscillatoire et sur la stabilisation de l'intensité de la tâche spéculaire. L'analyse des courbes obtenues et leur évolution permet de corréler les résultats avec le processus de croissance et de préciser les mécanismes qui interviennent dans l'épitaxie par jets moléculaires du $\mathrm{GaSb}$.
\end{abstract}

\begin{abstract}
We present in this paper a study by RHEED oscillations intensities of the growth of GaSb by M.B.E. For this analysis we have studied the effects of different parameters (substrate temperature, antimony and gallium flux, the growth interruption time) on oscillation regimes and on the stabilisation of the specular reflexion intensity. The correlation of these results with growth process permitted us to clarify the MBE mechanism of GaSb.
\end{abstract}

\section{Introduction.}

La technique de diffraction par réflexion d'électrons de grande énergie (RHEED) [pour Reflection High Energy Electron Diffraction] est devenue un moyen d'analyse essentiel pour le suivi in situ de la croissance des films monocristallins par la méthode d'épitaxie par jets moléculaires (EJM). Elle est utilisée pour contrôler l'arrangement cristallin des surfaces avant (substrat), pendant (interface de croissance) et après croissance (surface de la couche). Depuis quelques années, après les premières observations de Neave et al. en 1983 [1] relatives aux oscillations d'intensité RHEED, cette technique est devenue un moyen d'analyse du processus de croissance. Neave et al. [1] ont montré que la croissance couche après couche se traduit par des oscillations d'intensité de RHEED, dont l'atténuation est liée au démarrage d'une nouvelle couche avant que la couche sous-jacente ne soit complète. La période des oscillations d'intensité correspond au dépôt d'une double couche d'atomes ou monocouche moléculaire pour les matériaux III-V et peut ainsi servir à

Présenté au Colloque «Surfaces et Interfaces de Semiconducteurs » du Congrès de la S.F.P., Lyon, 1989. la mesure de la vitesse de croissance. Plus généralement la cinétique de l'intensité de la tache spéculaire permet une étude des mécanismes de croissance au travers de l'influence des conditions de croissance sur l'incorporation des éléments III et V [2].

Lorsque la croissance se fait couche par couche (type Franck Van Der Merwe), elle est dite bidimensionnelle (2D), un atome arrivant à la surface dispose de plusieurs types de sites d'incorporation [3] plus ou moins favorables énergétiquement. La probabilité de migration des atomes en surface croît avec la température. Pour une température de substrat suffisamment élevée, on favorise la mobilité et donc le développement des marches ou des terrasses. Par contre pour une température plus basse, lorsque la mobilité des atomes est insuffisante, l'incorporation sur des sites isolés ou nucléation 2D devient prépondérante. Le processus de croissance comprend la compétition entre ces deux mécanismes. Neave et al. [1] ont suggéré que les oscillations de l'intensité RHEED pouvaient être interprétées en terme de variation de la réflectivité de la surface de croissance par modification de la densité des îlots 2D en cours de croissance. Dans ce modèle, on attribue le minimum d'intensité à un minimum de réflectivité, qui correspond à un maximum de rugosité pour une 
densité maximale d'îlots 2D. Lorsque la taille des îlots croît, la réflectivité de la surface augmente jusqu'à donner un maximum d'intensité qui correspond dans le cas idéal à une couche complète. L'atténuation du phénomène oscillatoire est expliquée par une perte de la bidimensionnalité de la croissance dans le temps: la surface tend vers une rugosité moyenne attribuée à une distribution moyenne de terrasses et d'îlots $2 \mathrm{D}$ en équilibre thermodynamique. Alors la surface moyenne n'évolue plus et sa réflectivité est constante. Lors d'un arrêt de croissance, avec maintien de la température du substrat et du flux du matériau $V$, la surface se lisse par migration des atomes vers les marches et sa réflectivité tend vers celle d'une couche complète. Il convient cependant de séparer les mécanismes d'incorporation des cations et des anions. Le mécanisme d'incorporation de $\mathrm{Sb}$ (pour $\mathrm{GaSb}$ ) peut être rapproché du mécanisme d'incorporation de As (pour GaAs). Le flux de $\mathrm{Ga}$ est monoatomique avec un coefficient de collage voisin de 1, l'anion ( $\mathrm{Sb}$ ) est sous forme moléculaire $\left(\mathrm{Sb}_{2}, \mathrm{Sb}_{4}\right)$ et son adsorption n'a lieu que par interaction avec les atomes de $\mathrm{Ga}$ adsorbés. Il a été montré que les caractéristiques de l'incorporation du Ga déterminent le mode de croissance et que le phénomène cinétique fondamental devient la diffusion des atomes de $\mathrm{Ga}[4,5]$.

Les oscillations d'intensité RHEED en cours de croissance par EJM ont été observées pour différents matériaux : GaAs [6] (AlGa) As [1], (InGa) As [2], (InAl) As [7, 8], ZnSe et MnSe [9], Si [10], Ge [11]. Subanna et al. [12] ont récemment présenté des études d'oscillations RHEED en cours de croissance par EJM d'antimoniures (GaSb et AlSb) sur substrat de GaAs. Après avoir montré que l'importance du désaccord des paramètres de maille du substrat et de la couche $(=7 \%)$ nécessitait le dépôt préalable d'une couche tampon de $500 \AA$ de AlSb pour permettre une croissance $2 \mathrm{D}$ et l'observation d'oscillations d'intensité RHEED, ils ont étudié l'influence du rapport des flux (III/V) sur ces oscillations.

Nous présentons ici une étude des oscillations RHEED dont le but est l'analyse des mécanismes de croissance par EJM du GaSb sur substrat de GaSb. Le système $(\mathrm{AlGa}) \mathrm{Sb}$ a des propriétés physiques intéressantes et des possibilités d'applications potentielles en optoélectronique [13-17]. Les résultats que nous présentons sont une étape dans l'étude que nous faisons pour maîtriser l'élaboration de ces matériaux et de leurs interfaces.

\section{Technique expérimentale.}

Les expériences sont réalisées dans un bâti d'épitaxie par jets moléculaires, conçu au L.E.S.I.C. [18], et qui comporte trois enceintes (de transfert, d'analyse et de croissance). L'enceinte de croissance est équi- pée de cellules à effusion de $\mathrm{Ga}$ et $\mathrm{Sb}$ et comporte un système de diffraction d'électrons en incidence rasante (RHEED : $10 \mathrm{KeV}$ ) qui permet l'examen de la structure cristallographique de la surface pendant la croissance. Les substrats de GaSb $(001) \pm 0,5^{\circ}$ sur lesquels on fait l'épitaxie sont d'abord dégraissés, puis collés à l'indium sur un support en molybdène et ensuite nettoyés par un polissage mécano-chimique (brome-méthanol à $0,5 \%$ ). La préparation in situ destinée à obtenir une surface propre et ordonnée est ensuite effectuée dans l'enceinte de croissance par chauffage, à une température de l'ordre de $580{ }^{\circ} \mathrm{C}$ sous flux de $\mathrm{Sb}$. Le contrôle des flux de $\mathrm{Ga}$ et $\mathrm{Sb}$ est obtenu par l'intermédiaire de la régulation de la température des cellules. Un pyromètre infrarouge, situé face au substrat en position d'épitaxie, permet de déterminer la température du substrat.

Pour la mesure de l'intensité RHEED, nous avons installé sur le bâti d'épitaxie un dispositif classique où l'image d'un point du diagramme de diffraction est envoyée sur un photomultiplicateur par un système de lentilles et une fibre optique [19]. Le signal fourni par le détecteur est enregistré en fonction du temps. Les études sont faites après croissance d'une couche tampon de quelques centaines d'angströms, destinée à éliminer les imperfections de la surface du substrat liées au polissage. Deux échantillons sont disposés sur le porte-échantillon pour permettre l'examen des diagrammes de RHEED selon les deux azimute [110] et [1̄10] du plan (001) sous un angle d'incidence de l'ordre du degré. Nous avons toujours enregistré l'intensité de la tâche spéculaire dans l'azimut [1110] où l'on observe la surstructure $1 / 3$ de la surface de croissance. Certains détails des oscillations de la tâche spéculaire étant fonction de l'angle d'incidence [20], nous avons comparé les mesures effectuées avec un même angle d'incidence.

L'évolution avec le temps de l'intensité de la tache spéculaire présente, d'une manière générale (Fig. 1), les caractéristiques suivantes :

- Stabilité de l'intensité pour une surface maintenue à température constante sous flux de Sb (Intensité statique notée $I_{\mathrm{s}}$ ).

- Oscillation de l'intensité au début de croissance $\left(t \geqslant t_{c}\right)$.

- Par amortissement du phénomène oscillatoire, l'intensité tend vers une valeur constante appelée intensité dynamique notée $I_{\mathrm{d}}$.

- A la fermeture de la cellule de Ga $\left(t=t_{a}\right)$, $I$ retourne vers la valeur $I_{\mathrm{s}}$, c'est le phénomène de récupération de l'intensité à l'arrêt de croissance.

\section{Résultats.}

L'étude de l'évolution de l'intensité de la tache spéculaire du diagramme de diffraction a été effectuée en faisant varier les conditions de croissance. 


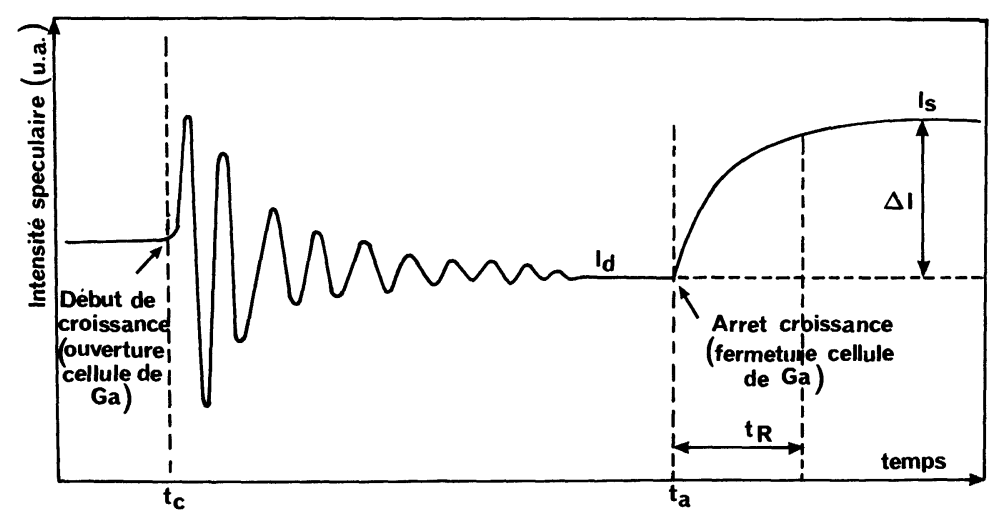

Fig. 1. - Représentation schématique de l'intensité de la tache spéculaire.

[Schematic representation of the specular spot intensity.]

Nous résumons cette étude en présentant les effets liés à la température du substrat, aux flux des éléments III et $\mathrm{V}$, et enfin nous examinons la récupération de l'intensité consécutive à un arrêt de croissance.

Dans le domaine d'investigation des paramètres de croissance, en cours de croissance et pendant les arrêts de croissance sous flux d'antimoine, nous avons observé le même diagramme de diffraction correspondant à une sur-structure $(1 \times 3)$ de la surface (001) du GaSb.

EFFET DE LA TEMPÉRATURE DU SUBSTRAT. - Nous avons représenté sur la figure 2 l'évolution des diagrammes d'oscillations avec la température du substrat dans le domaine $440-490^{\circ} \mathrm{C}$.

Nous avons noté $I_{\mathrm{s} i}(i=0$ à 6$)$ les intensités lorsque la croissance était arrêtée (cellule Ga fermée, cellule $\mathrm{Sb}$ ouverte), la surface stabilisée et avant l'ouverture de la cellule de Ga. Lors d'un démarrage d'une croissance, nous observons au début des oscillations qui s'amortissent dans le temps, l'intensité de la tache spéculaire tendant vers la valeur $I_{\mathrm{d} i}$. On remarque que les intensités $I_{\mathrm{s}}$ et $I_{\mathrm{d}}$ croissent avec la température. L'évolution relative de ces grandeurs, ainsi que le signe de la variation de $I$ quand on ouvre le cache de $\mathrm{Ga}$, permettent de distinguer trois catégories de courbes d'oscillations comme lors de la croissance de GaAs par EJM [21] :

- Pour la catégorie $(I)$, observée à $440{ }^{\circ} \mathrm{C}$, les oscillations débutent par un maximum avec $I=I_{\mathrm{d}}-I_{\mathrm{s}}$ positif.

- Pour la catégorie (II), observée de 445 à $460{ }^{\circ} \mathrm{C}, I$ croît comme précédemment à l'ouverture, mais moins fortement et $\Delta I$ est négatif.

- La catégorie (III) correspond à la disparition du premier maximum et est observée pour $T_{\mathrm{s}} \geqslant 470^{\circ}$. Au-delà de $T_{\mathrm{s}}=495^{\circ}$, les oscillations deviennent difficiles à observer.
Il faut noter que la vitesse de croissance $\left(V_{\mathrm{GaSb}}\right)$, déduite de la période des oscillations, décroît lorsque $T_{\mathrm{s}}$ passe de $490^{\circ}$ à $440{ }^{\circ} \mathrm{C}$. On mesure $\Delta V / V=-7 \%$ et $-3 \%$ pour des pressions d'antimoine dans l'enceinte respectivement de $5 \times 10^{-9}$ et $2 \times 10^{-9}$ Torr.

EFfeT DU FluX DE Sb. - Pour cette étude, la température du creuset de $\mathrm{Ga}$ et la température du substrat sont maintenues constantes. La variation du flux d'antimoine est repérée par la pression dans l'enceinte qui est proportionnelle à la pression partielle de Sb. L'augmentation du flux de Sb (Fig. 3) induit un passage progressif de la catégorie (II) à la catégorie (I) et une décroissance des intensités spéculaires $I_{\mathrm{s}}$ et $I_{\mathrm{d}}$.

La mesure de la vitesse de croissance, à partir de la période des oscillations, montre que l'accroissement du flux de $\mathrm{Sb}$ a pour effet de diminuer la vitesse de croissance de $\mathrm{GaSb}$ (quelques \% pour un rapport 1 à 4 des flux).

EFFET DU FLUX DE Ga. - Cette étude est effectuée (Fig. 4) pour $T_{\mathrm{s}}=445^{\circ} \mathrm{C}$ et avec une pression dans l'enceinte de $2,3 \times 10^{-9}$ Torr. Pour une faible vitesse de croissance, c'est-à-dire avec un faible flux de $\mathrm{Ga}$, la croissance est du type (I), elle devient du type (II) lorsque le flux de $\mathrm{Ga}$ augmente. De cette étude, on en déduit la courbe d'étalonnage qui donne la vitesse de croissance du GaSb en fonction de la température du creuset de $\mathrm{Ga}$ et la valeur de l'énergie d'activation d'évaporation du $\mathrm{Ga}\left(E_{\mathrm{Ga}}=2,6 \mathrm{eV}\right)$ en accord avec les données thermodynamiques de Honig et Kramer [22].

RÉCUPÉRATION DE L'INTENSITÉ À L'ARRÊT DE CROISSANCE. - Nous avons reporté sur la partie gauche de la figure 5 des courbes de retour vers l'intensité statique qui montrent que la récupération 


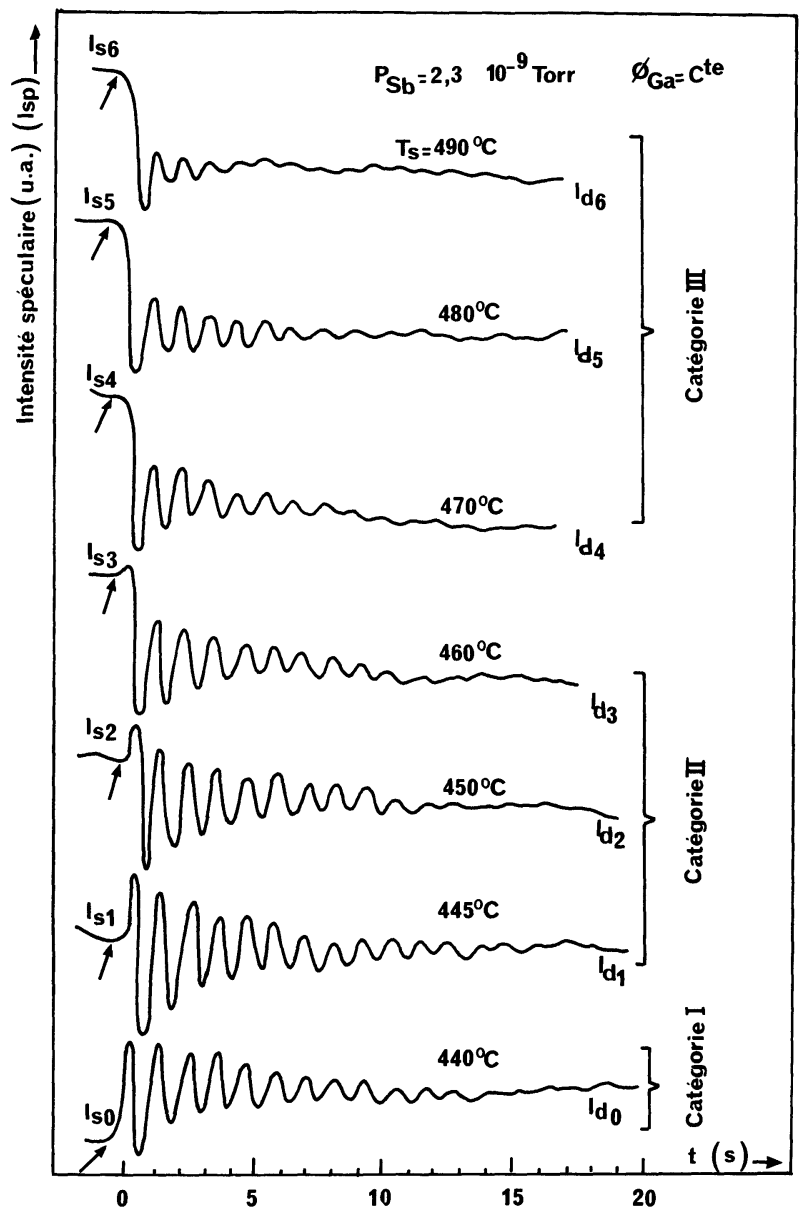

Fig. 2. - Evolution des oscillations RHEED en fonction de la température du substrat $\left(T_{\mathrm{s}}\right)$ à $P_{\mathrm{Sb}}=2,3 \times 10^{-9}$ Torr et $\phi_{\mathrm{Ga}}$ constant. La flèche indique le début de croissance. Dans le domaine $440-500{ }^{\circ} \mathrm{C}$, à température constante la variation de $I_{\mathrm{sp}}\left(I_{\mathrm{s}}-I_{\mathrm{d}}\right)$ liée à la croissance est d'environ $\pm 10 \%$. D'autre part, $I_{\mathrm{s}}$ croît avec la température à raison de 10 à $20 \%$ par $10^{\circ} \mathrm{C}$.

[Evolution of the RHEED oscillation during the growth versus the substrate temperature $T_{\mathrm{s}}$ at $P_{\mathrm{Sb}}=2.3 \times 10^{-9}$ Torr and constant flux of $\mathrm{Ga}$. The arrow indicates the start of the growth. In the $440-500{ }^{\circ} \mathrm{C}$ temperature range, at constant temperature the $\left(I_{\mathrm{s}}-I_{\mathrm{d}}\right)$ variation of $I_{\mathrm{sp}}$ during the growth is about $\pm 10 \%$. Furthermore, $I_{\mathrm{sp}}$ increases when the substrate temperature $T_{\mathrm{s}}$ increases $\left(I=+10\right.$ to $+20 \%$ for $\left.T_{\mathrm{s}}=+10{ }^{\circ} \mathrm{C}\right)$.]

d'intensité par l'arrêt de croissance diffère avec les conditions de croissance. Il apparaît également des courbes de récupération caractéristiques selon la catégorie de croissance I, II ou III. Pour une analyse plus fine de ce phénomène, nous avons effectué une étude détaillée, ayant pour but la caractérisation de la surface à différents stades de la récupération. Cela a été réalisé en effectuant des redémarrages de croissance à différents instants $t_{1}, t_{2}, t_{3} \ldots$ après arrêt de la croissance ; avant chaque arrêt de croissance on attend la stabilité de l'intensité dynamique de la

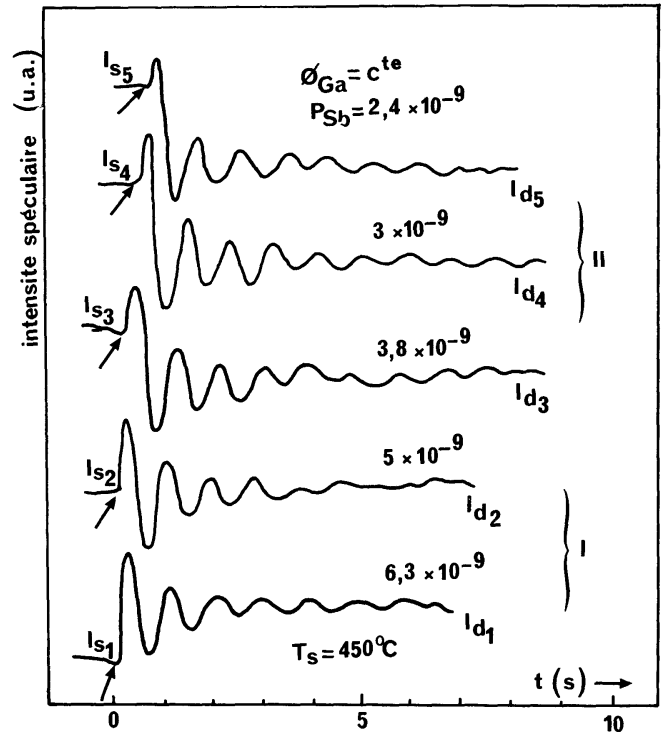

Fig. 3. - Evolution des oscillations RHEED en fonction du flux de Sb à $T_{\mathrm{s}}=450{ }^{\circ} \mathrm{C}, V_{\mathrm{GaSb}}=1,10 \mu \mathrm{m} / \mathrm{h}$. La flèche indique le début de croissance.

[RHEED oscillations during growth as a function of $\mathrm{Sb}$ flux with $T_{\mathrm{s}}=450{ }^{\circ} \mathrm{C}$ and $V_{\mathrm{Gasb}}=1.10 \mu \mathrm{m} / \mathrm{h}$. The arrow indicates the start of the growth.]

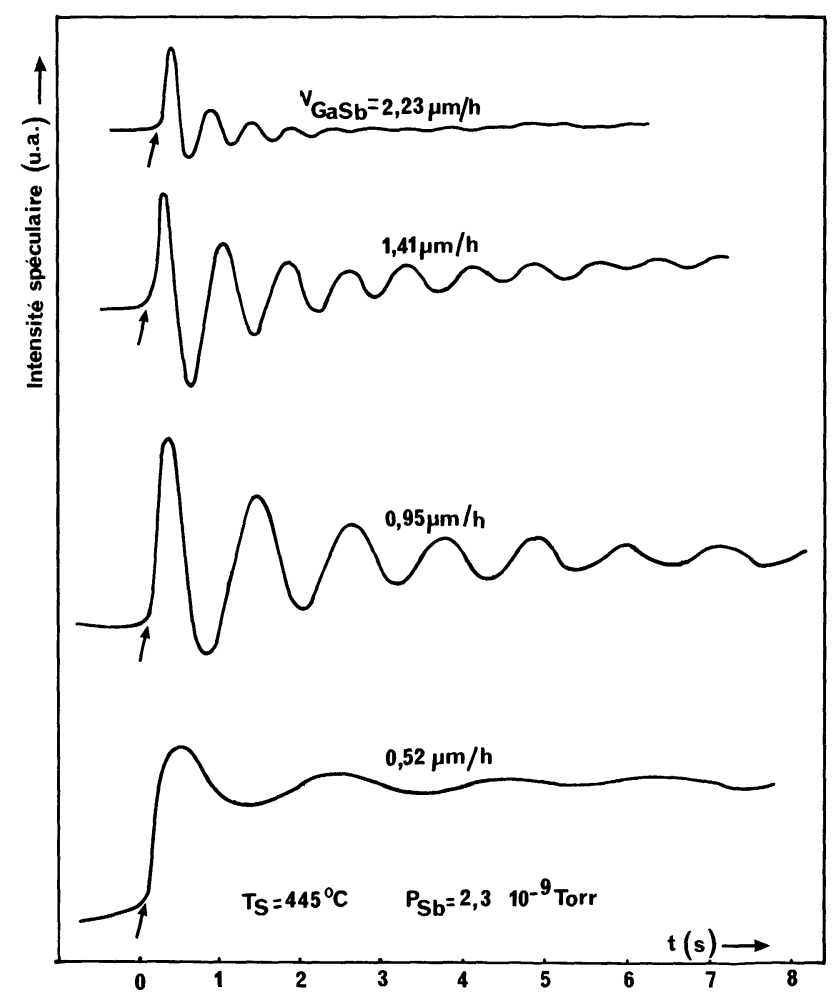

Fig. 4. - Evolution des oscillations RHEED en fonction du flux de Ga à $T_{\mathrm{s}}=445^{\circ} \mathrm{C}$ et $P_{\mathrm{Sb}}=2,3 \times 10^{-9}$ Torr. La flèche indique le début de croissance.

[Evolution of the RHEED oscillations during the growth for different values of $\mathrm{Ga}$ flux with $T_{\mathrm{s}}=445^{\circ} \mathrm{C}$ and $P_{\mathrm{Sb}}=2.3 \times 10^{-9}$ Torr. The arrow indicates the start of the growth.] 
croissance. Sur la partie droite de la figure 5, nous avons reporté des diagrammes d'oscillations RHEED correspondant à des redémarrages de croissance $2 \mathrm{~s}, 5 \mathrm{~s}, \ldots, 2 \mathrm{mn}$, après arrêt de la croissance. On remarque des évolutions dans les courbes très importantes, et en particulier que le phénomène oscillatoire est d'autant plus important que le temps de récupération de la surface (temps écoulé après l'arrêt de croissance) est grand. On observe que, dans les conditions de croissance correspondant à la figure 5b, un temps d'arrêt supérieur à $30 \mathrm{~s}$ est nécessaire pour obtenir le diagramme d'oscillations de catégorie (II) caractéristique des conditions de croissance.

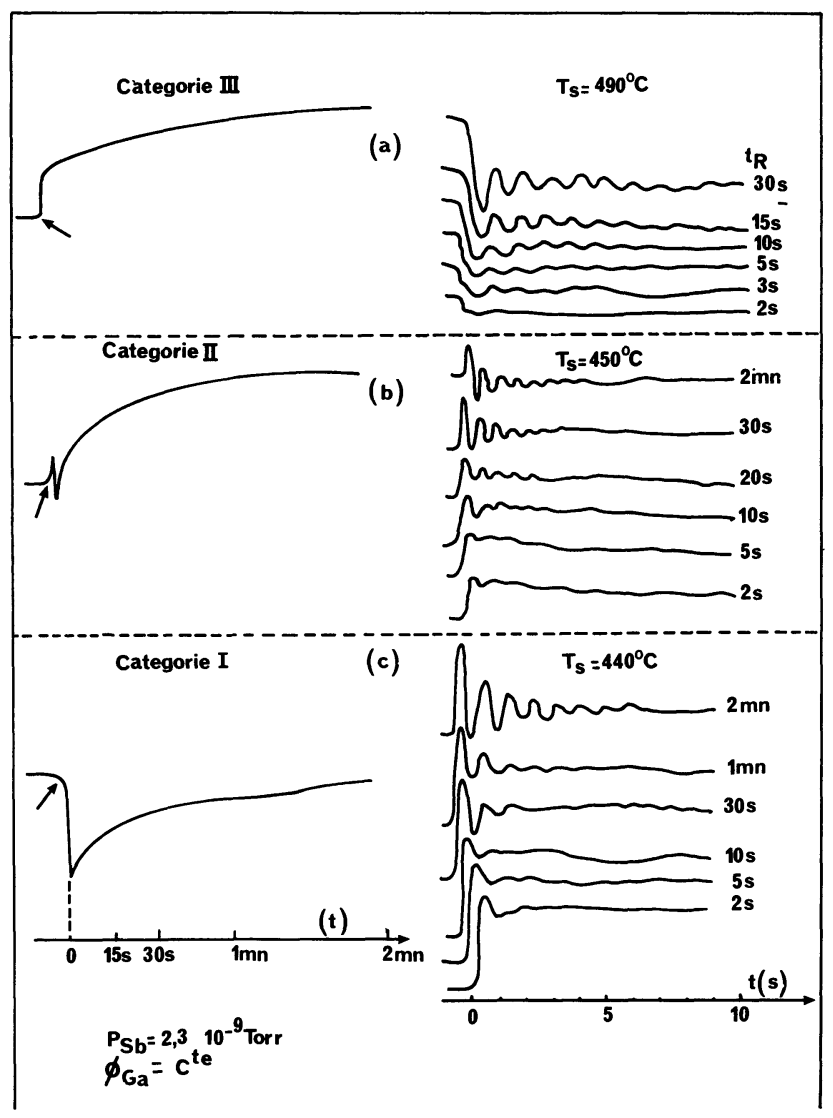

Fig. 5. - Courbes de récupération de l'intensité de la tache spéculaire en fonction du temps d'arrêt de croissance pour différentes températures d'épitaxie : a) $T_{\mathrm{s}}=490{ }^{\circ} \mathrm{C}$, b) $T_{\mathrm{s}}=450{ }^{\circ} \mathrm{C}$, c) $T_{\mathrm{s}}=440{ }^{\circ} \mathrm{C}$. Oscillations RHEED obtenues par démarrage de croissance selon le temps d'arrêt.

[Specular intensity stabilization after growth interruption for different temperatures of epitaxy : a) $T_{\mathrm{s}}=490{ }^{\circ} \mathrm{C}, b$ ) $T_{\mathrm{s}}=450{ }^{\circ} \mathrm{C}$, c) $T_{\mathrm{s}}=440{ }^{\circ} \mathrm{C}$. RHEED oscillations obtained by growth starting after different times of growth interruption.]

\section{Discussion des résultats.}

Chacun des paramètres de croissance que nous avons étudiés $\left(T_{\mathrm{s}}, \phi_{\mathrm{Sb}}, \phi_{\mathrm{Ga}}\right)$ a pour effet de modifier le taux de recouvrement de $\mathrm{Sb}$ et la migration des atomes de $\mathrm{Ga}$ à la surface de la façon suivante :

- Par accroissement de $T_{\mathrm{s}}$ on facilite la diffusion des atomes de $\mathrm{Ga}$ ainsi que la désorption des atomes de $\mathrm{Sb}$ de la surface : Le recouvrement en $\mathrm{Sb}$ diminue et la mobilité des atomes de Ga croît.

- Par accroissement de flux de $\mathrm{Sb}$, avec l'augmentation du taux de recouvrement de la surface par Sb on doit s'attendre à une diminution de la mobilité des atomes de $\mathrm{Ga}$ [23].

- Par accroissement du flux de Ga, la mobilité des atomes de Ga à la surface décroît et le recouvrement en atomes de $\mathrm{Sb}$ devient relativement plus faible.

On sait que d'après le modèle de Neave et al. $[1,5]$, la migration des atomes $\mathrm{Ga}$ à la surface, détermine le processus de croissance (taille et densité des îlots). Les modifications de rugosité ou de réflectivité de la surface, avant, pendant, ou après croissance, sont donc liées d'une manière interdépendante à la concentration et à la diffusion des espèces à la surface. Les trois catégories de courbes d'oscillation d'intensité de la tache spéculaire que nous avons observées en faisant varier $T_{\mathrm{s}}$ (Fig. 2) traduisent des surfaces initiales différentes et des conditions de croissance différentes. La surface initiale est la surface maintenue à la température $T_{\mathrm{s}}$ sous flux de $\mathrm{Sb}$ constant, le flux de Ga étant interrompu. Lorsque $T_{\mathrm{s}}$ croît, l'intensité spéculaire statique $\left(I_{\mathrm{s}}\right)$ croît par un meilleur lissage de la surface résultant d'une plus grande mobilité des atomes. Dans le cas d'une croissance de type I à $T_{\mathrm{s}}$ faible, le fait que l'intensité en dynamique soit supérieure à l'intensité en statique, montre que l'apport du flux de $\mathrm{Ga}$ diminue la rugosité de la surface par la formation d'îlots de plus grande dimension. Le premier maximum résulte du déclenchement de ce processus dès l'arrivée des atomes de Ga. Lorsqu'on augmente $T_{\mathrm{s}}$, l'intensité statique de la surface initiale $\left(I_{\mathrm{s}}\right)$ croît plus vite que l'intensité dynamique $\left(I_{\mathrm{d}}\right)$ : d'où $\Delta I=I_{\mathrm{d}}-I_{\mathrm{s}}$ diminue et devient négatif pour les catégories II et III. L'augmentation de la valeur de l'intensité dynamique $\left(I_{\mathrm{dIII}}>I_{\mathrm{dII}}>I_{\mathrm{dI}}\right)$ indique à nouveau un meilleur lissage de la surface par élargissement des terrasses en cours de croissance, lorsque $T_{\mathrm{s}}$ croît. Autrement dit, l'amélioration du lissage de la surface initiale, par accroissement de $T_{\mathrm{s}}$, est plus importante que celle résultant de l'apport du flux de Ga. Ceci peut être vu comme le résultat de la compétition entre îlots et terrasses. La perte des oscillations, au démarrage de la croissance, lorsque $T_{\mathrm{s}}>500{ }^{\circ} \mathrm{C}$, indique que le régime dynamique est obtenu instantanément par la très grande mobilité des atomes de 
Ga et donc le processus de formation de terrasses à la surface est prépondérant.

La diminution de $I_{\mathrm{s}}$ et $I_{\mathrm{d}}$ et l'accroissement du premier maximum, lorsque le flux de $\mathrm{Sb}$ augmente, sont équivalents en terme de rugosité à une diminution de $T_{s}$. On peut les attribuer à une plus forte adsorption d'atomes de $\mathrm{Sb}$ à la surface qui entraîne une baisse de la mobilité des atomes de $\mathrm{Ga}$, ce qui facilite la formation d'îlots et donne un accroissement de la rugosité.

La transition d'une catégorie à une autre n'est pas très sensible à la modification du flux de $\mathrm{Ga}$. A faible flux de $\mathrm{Ga}$ et donc à faible vitesse de croissance la mobilité des atomes de $\mathrm{Ga}$ est plus grande et le lissage est meilleur par la formation de terrasses plus larges. La légère diminution de la vitesse de croissance (de l'ordre de 5-7\%), lorsque la température du substrat est abaissée ou lorsqu'on augmente le flux de $\mathrm{Sb}$, peut être attribuée à la diminution de la mobilité des atomes de Ga lorsque la surface s'enrichit en élément V (23-24). Dans ces conditions de croissance, on peut formuler l'hypothèse que la formation de domaines tridimensionnels est facilitée, et ainsi par l'observation des oscillations on mesure une diminution de la vitesse de croissance bidimensionnelle.

En première analyse, les courbes de récupération d'intensité présentent un régime rapide et un régime lent. Dans tous les cas de croissance, la récupération lente de l'intensité est liée au phénomène de lissage de la surface, ce qui explique que les redémarrages de croissance, après un temps de récupération important, produisent des oscillations de plus grande amplitude. On observe quelquefois (Fig. 5b) au redémarrage, des courbes d'oscillations qui ne correspondent pas à la catégorie caractéristique des conditions de croissance. Ceci s'explique par le fait qu'à chaque instant de la récupération correspond une surface initiale caractéristique pour le redémarrage de croissance qui suit.
Le régime rapide qui précède le phénomène lent de récupération traduit un effet consécutif à la suppression du flux de $\mathrm{Ga}$. A basse température (440), l'adsorption de $\mathrm{Sb}$ en surface explique la diminution de l'intensité par accroissement de la rugosité, au contraire, à haute température $\left(490^{\circ} \mathrm{C}\right)$, en l'absence du flux de $\mathrm{Ga}$, la désorption de $\mathrm{Sb}$ de la surface améliore très rapidement le lissage de la surface.

Il est remarquable qu'à la température intermédiaire de $450^{\circ}$, on observe une augmentation (lissage rapide), puis une diminution de l'intensité par l'adsorption de $\mathrm{Sb}$, ce qui indiquerait un retard au collage des atomes de $\mathrm{Sb}$ à la surface.

\section{Conclusion.}

L'étude de l'évolution de l'intensité de la tache spéculaire du diagramme RHEED nous a permis d'analyser la contribution des différents paramètres de croissance de GaSb par EJM. Nous avons interprété les résultats obtenus en nous appuyant sur le modèle de la croissance bidimensionnelle et en considérant que les modifications d'intensité sont liées à des modifications de réflectivité de la surface.

Nous avons montré que l'intensité de la tache spéculaire dépend fortement des différents paramètres (température de substrat, flux de $\mathrm{Ga}$ et de $\mathrm{Sb}$, temps de récupération).

Nous avons attribué ce phénomène à une évolution de la rugosité de surface en faisant intervenir des terrasses et des îlots $2 \mathrm{D}$ dont la formation et l'évolution ont été expliquées en prenant en compte les mécanismes d'adsorption-désorption et la mobilité des atomes en surface.

Nous avons montré que les flux de $\mathrm{Ga}$ et $\mathrm{Sb}$ et la température du substrat jouaient un rôle, la contribution de la température étant cependant beaucoup plus importante.

\section{Bibliographie}

[1] Neave J. H., Joyce B. A., Dobson P. J. and NorTON N., Appl. Phys. A 31 (1983) 1.

[2] Lewis B. F., Lee T. C., Grunthaner F. J., MADHUKAR A., FERNANDEZ R. and MASERJIAN J., J. Vac. Sci. Technol. B 2 (1984) 419.

[3] Burton W. B., Cabrera N. and Franck F. C., Phil. Trans. R. Soc. A 243 (1951) 299.

[4] Foxon C. T. and Joyce B. A., Surf. Sci. 50 (1975) 434.

[5] Neave J. H., Dobson P. J., JoyCe B. A. and Zhang J., Appl. Phys. Lett. 47 (1985) 100.

[6] Massies J., $7^{\mathrm{e}}$ Ecole d'Eté Méditerranéenne (Toulouse, 1988).
[7] Berger P. R., Bhattacharya P. K. and Singh J., J. Appl. Phys. 61 (1987) 2856.

[8] Lievin J. L. and Fonstad C. G., Appl. Phys. Lett. 51 (1987) 1173.

[9] Gunshor R. L., Kolodziejski L. A., Melloch M. R., VAziri M., Choi C. and Otsuka N., Appl. Phys. Lett. 50 (1987) 200.

[10] Sakamoto, Kawai N. J., Nakagawa T., Ohta K. and Kojima T., Appl. Phys. Lett. 47 (1985) 617.

[11] Aarts J., Gerits W. M. and Larsen P. K., Appl. Phys. Lett. 48 (1986) 931.

[12] Subbanna S., Gaines J., Tuttle G., Kroemer H., Chalmers S. and English J. H., J. Vac. Sci. Technol. B 7 (1989) 289. 
[13] OHMORI Y., TARUCha S., Horikoshi Y. and OKAмото H., Jpn. J. Appl. Phys. 23 (1984) L93.

[14] Chiu T. H., Tsang W. T., Ditzenberger J. A. and VAN Der Ziel J. P., Appl. Phys. Lett. 49 (1986) 1051.

[15] Cebulla U., Trankle G., Ziem U., Forchel A., Griffiths G., Kroemer H. and SubbanNa S., Phys. Rev. B 37 (1988) 6278.

[16] Shen H., Hang Z., Leng Jing, Pollak F. H., Chang L. L., WANG W. I. and Esaki L., Superlattices microstruct. 5 (1989) 591.

[17] Raisin C., Lassabatere L., Alibert C., Girault B., Abdel-Fattah G. and Voisin P., Solid. State Comm. 61 (1987) 17.
[18] Raisin C., Saguintaah B., Tegmousse H., Lassabatere L., Girault B. and Alibert C., Ann. Télécomm. 41 (1986) 50.

[19] FoxoN C. T., Heterojunction and semiconductor superlattices. G. Allan et al. (Springer-Verlag) 1986, p. 216.

[20] Madhukar A., Chen P., Voillot F., Thomsen M., Kim J. Y., TANG W. C. et Ghaisas S. V., $J$. Cryst. Growth 81 (1987) 26.

[21] Charasse M. N., Thèse (Paris, 1985).

[22] Honig E. H. and Kramer D. A., R.C.A. Rev. 30 (1969) 285.

[23] Van Hove J. M. and Cohen P. I., J. Cryst. Growth 81 (1987) 13.

[24] Turco F., Thèse (Grenoble, 1988). 